\title{
Bilateral sixth nerve palsy as a manifestation of Wernicke's encephalopathy in a patient with refractory vomiting
}

This article was published in the following Dove Press journal:

Eye and Brain

3 July 2010

Number of times this article has been viewed

\author{
Domenico Porfido' \\ Silvana Guerriero' \\ Giovanni Giancipoli' \\ Michele Vetrugno' \\ Velia Lefons ${ }^{2}$ \\ Franca Dicuonzo² \\ 'Department of Ophthalmology, \\ ${ }^{2}$ Department of Neuroradiology, \\ University of Bari, Italy
}

Correspondence: Domenico Porfido Università degli Studi di Bari,

Piazza G. Cesare, II, 70124 Bari, Italy

Tel $+393389 \mid 49579$

$\mathrm{Fax}+390805478918$

Email porfidodomenico@hotmail.com
Purpose: To report a case of Wernicke's encephalopathy in a nonalcoholic woman with secondary hypoadrenalism.

Methods: A 58-year-old Italian woman developed Wernicke's syndrome secondary to recurrent vomiting due to secondary hypoadrenalism.

Results: Recurrent vomiting and resulting malnutrition caused a depletion of the patient's body stores of thiamine and the development of mental confusion and an oculomotor deficit. On the diagnostic suspicion of encephalitis in this patient with immunosuppression due to prolonged cortisone-based therapy, she underwent magnetic resonance imaging, which showed typical bilateral abnormal lesions pathognomonic of Wernicke's encephalopathy. The patient improved after parenteral administration of vitamin B1.

Conclusions: Wernicke's encephalopathy must be regarded as a medical emergency that should be suspected in all cases presenting with a sudden deficit or lack of coordination of the ocular movements associated with ataxia and general degeneration of the mental faculties, in patients who have suffered recurrent vomiting or malnutrition for different reasons.

Keywords: hypoadrenalism, oculomotor abnormalities, Wernicke's syndrome

\section{Introduction}

Wernicke's syndrome is an acute neurological disorder due to a thiamine deficiency. Thiamine pyrophosphate (TPP) is a co-enzyme required for linking glycolysis to the Krebs cycle in the aerobic metabolism of glucose, as well as in several other steps in carbohydrate metabolism. One of the consequences of a thiamine deficiency is its negative effect on those cells that rely heavily on the supply of glucose for energy, eg, brain cells. It is commonly associated with chronic alcoholism but also with starvation, persistent vomiting, and systemic diseases such as cancer, liver failure, disseminated tuberculosis, and uraemia. Iatrogenic causes include inadequateparenteral nutrition or hemodialysis.

Wernicke's syndrome is characterized by three symptoms: an overall confusional state, disturbances of gait, and oculomotor abnormalities. ${ }^{1}$ However, some patients may not exhibit this triad. The diagnosis is then based on the clinical suspicion and rapid reversibility of symptoms after the administration of thiamine, and on the pathognomonic magnetic resonance imaging (MRI).

We report an unusual case of Wernicke's encephalopathy in a nonalcoholic woman with secondary hypoadrenalism. 


\section{Case report}

A 58-year-old Italian woman presented to the gastroenterological department complaining of recurrent vomiting and malnutrition. On admission the patient seemed responsive, conscious, space-time oriented, but asthenic; she had a plethoric face with dry skin and slight edema of the eyelids and of the lower limbs. Physical examination revealed a normal heart rate of 72 beats $/ \mathrm{min}$; blood pressure was 130/90 $\mathrm{mmHg}$, body temperature was $37.8^{\circ} \mathrm{C}$ and there was no thyroid enlargement or palpable lymph nodes. Blood examination showed a normocytic anemia with lymphocytosis, elevated levels of plasma creatinine $120 \mu \mathrm{M}$ (normal range: 63-105 $\mu \mathrm{M}$ ), plasma sodium was slightly low: $134 \mathrm{mM}$ (normal range: 136-146 $\mathrm{mM}$ ), and plasma potassium was slightly elevated: $5.3 \mathrm{mM}$ (normal range $3.5-5 \mathrm{mM}$ ). Serum phosphate was $3.1 \mathrm{mg} / \mathrm{dL}$ (normal range $2.5-4.5 \mathrm{mg} / \mathrm{dL}$ ).

Her past medical history included chronic use of corticosteroids (betamethasone $1-2 \mathrm{mg}$ per day for five years) for hip osteoarthritis, followed by a sudden suspension of the therapy about two months before, without gradually decreasing the dosage. A secondary hypoadrenalism due to the sudden reduction of the steroidal therapy was diagnosed on the morning plasma cortisol levels of $3 \mathrm{ng} / \mathrm{mL}$ (normal range morning: 9-23 ng/mL, afternoon: 2-10 ng/mL) and corticotropine (ACTH) of $82 \mathrm{pg} / \mathrm{mL}$ (normal range: $5-77 \mathrm{pg} / \mathrm{mL}$ ). The patient was administered replacement therapy (hydrocortisone $50 \mathrm{mg} \times 3$ /day for 3 days, then $25 \mathrm{mg} \times 3$ ).

Despite this new therapy, 20 days after admission the patient showed weakness, malnutrition, space-time confusion, and persistent vomiting, and so parenteral nutrition was started. Her blood pressure was 150/90 and she was unable to abduct the eyes due to a sixth nerve (VI) cranial nerves deficit.

She became confused, disorientated, with memory loss, and her verbal responses were babbling and distorted. She was also found to have a complete horizontal conjugate gaze palsy. The patient did not complain of diplopia only because she was too confused to be aware of it. Fundus examination showed hyperemic and slightly edematous optic discs with normal vessels.

Because of these last symptoms, she was sent to the Department of Infectious Diseases on the diagnostic suspicion of encephalitis, together with immunosuppression due to prolonged cortisone-based therapy. She was empirically given cephtriaxone, vancomycin, and acyclovir.

A cerebrospinal tap was performed but gram stain, cell counts, cultures, and serologies, including arbour virus and herpes virus, were negative. All antibiotics were stopped immediately after the spinal fluid cultures were reported negative.
She underwent computed tomography (CT) of the brain and CT angiography, which did not show pathological findings, as well as electroencephalogram (EEG), which revealed diffuse slowing of the brain activity, but no epileptiform activity.

We then performed MRI of the brain, which showed typical bilateral abnormal lesions in the mamillary bodies, in the medial thalami and periaqueductal regions of the midbrain, on $\mathrm{T}_{2}$-weighted and fluid-attenuated inversion recovery (FLAIR) sequences (Figures 1-3). In addition to conventional MRI sequences, we performed diffusion-weighted imaging (DWI) (Figure 4), which revealed the abnormal signal changes more distinctly than the conventional sequences had done. Since in Wernicke's encephalopathy the lesion consists of brain vasogenic edema, DWI is useful for detecting pathological changes in this encephalopathy. DWI confirmed and underlined the symmetrical pathological thalamic and midbrain signal hyper intensities. An apparent diffusion coefficient (ADC) map also showed slightly high intensity in the same regions, suggestive of vasogenic edema. Apart from movement artifacts, none of the lesions were enhanced after the injection of contrast medium on $\mathrm{T}_{1}$-weighted axial images. These findings are pathognomonic of Wernicke's encephalophathy, as previously reported. ${ }^{2-4}$

We diagnosed Wernicke's syndrome and gave the patient $100 \mathrm{mg} /$ day of vitamin B1 by parenteral administration. After about 48 hours the woman's condition improved rapidly and she resumed consciousness and space-time orientation. The abduction defect improved quickly after the administration of intravenous high doses of thiamine, while the mental status improved more slowly and the muscular weakness slowly reduced.

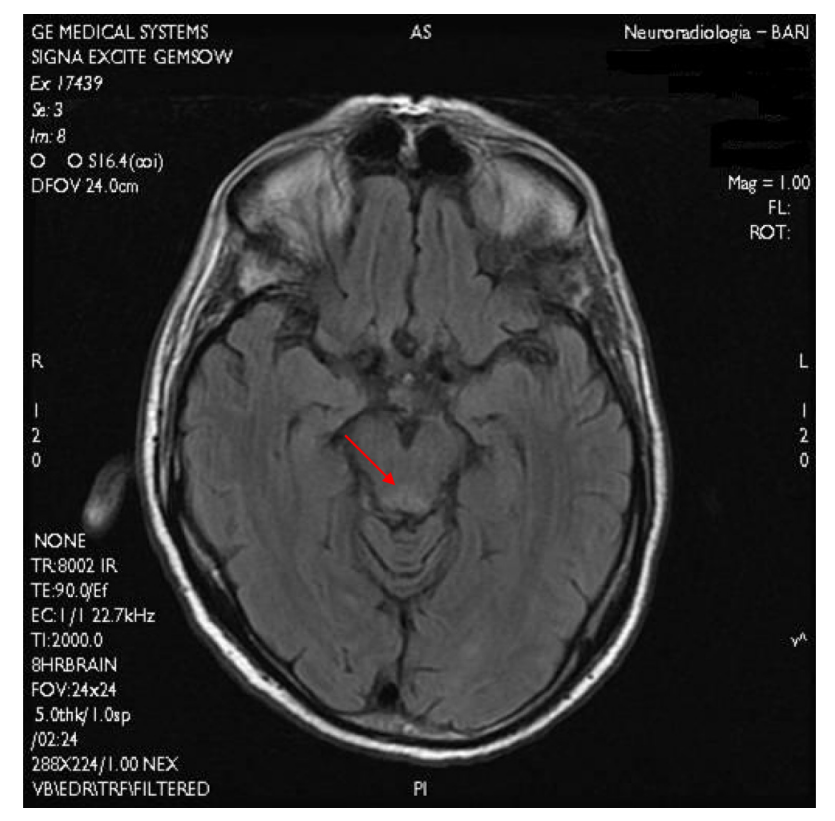

Figure I Magnetic resonance imaging: FSET2-FLAIR sequence, axial scan. Hyperintense lesion in the periacqueductal gray matter. 


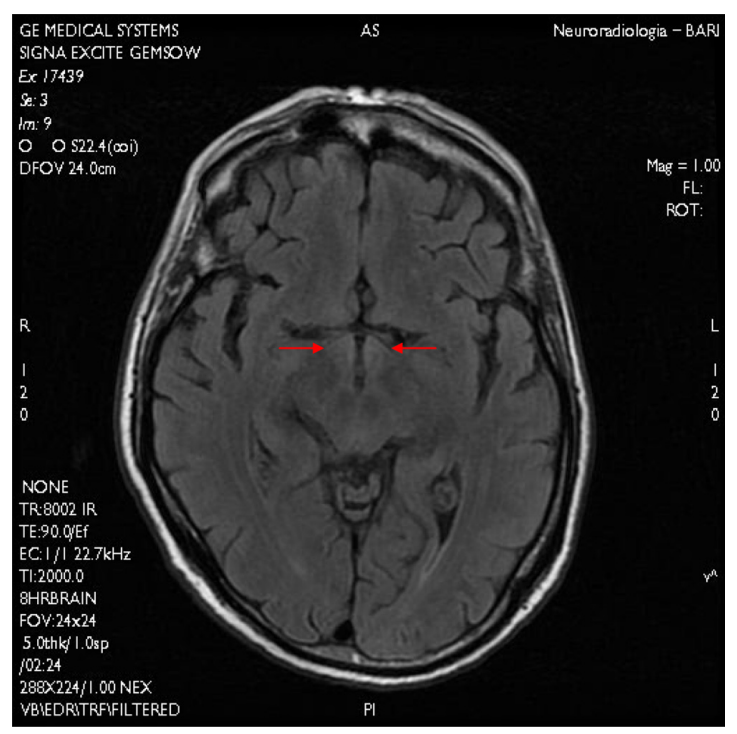

Figure 2 Magnetic resonance imaging: FSET2-FLAIR sequence, axial scan. Hyperintense lesions in the mammillary bodies.

\section{Discussion}

Wernicke's encephalopathy is a common but predictable disorder caused by a deficiency of thiamine. Thiamine pyrophosphate is a coenzyme required for linking glycolysis to the Krebs cycle in the aerobic metabolism of glucose, as well as in several other steps in carbohydrate metabolism. ${ }^{5}$

A thiamine deficiency induces neurological manifestations, peripheral, cerebral, cardiovascular, and gastrointestinal disturbances. ${ }^{6}$ Thiamine can be found in great quantities in animal and vegetable ingredients, such as pork meat, cereals, pulses, and in beer yeast. It is also produced by the intestinal flora; daily intake should be at least $1.2 \mathrm{mg}$. Body stores of

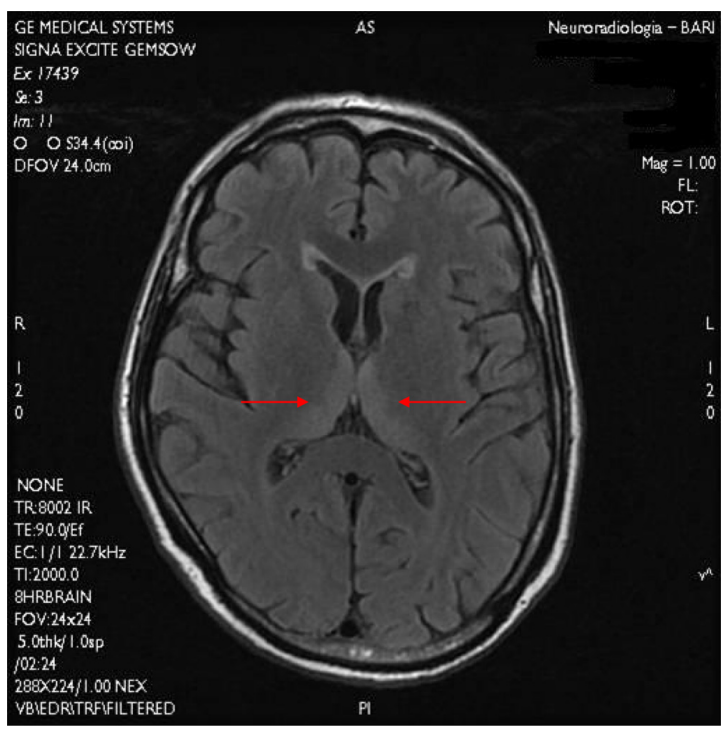

Figure 3 Magnetic resonance imaging: FSET2-FLAIR sequence, axial scan. Symmetrical hyperintense lesions in the medial thalami.

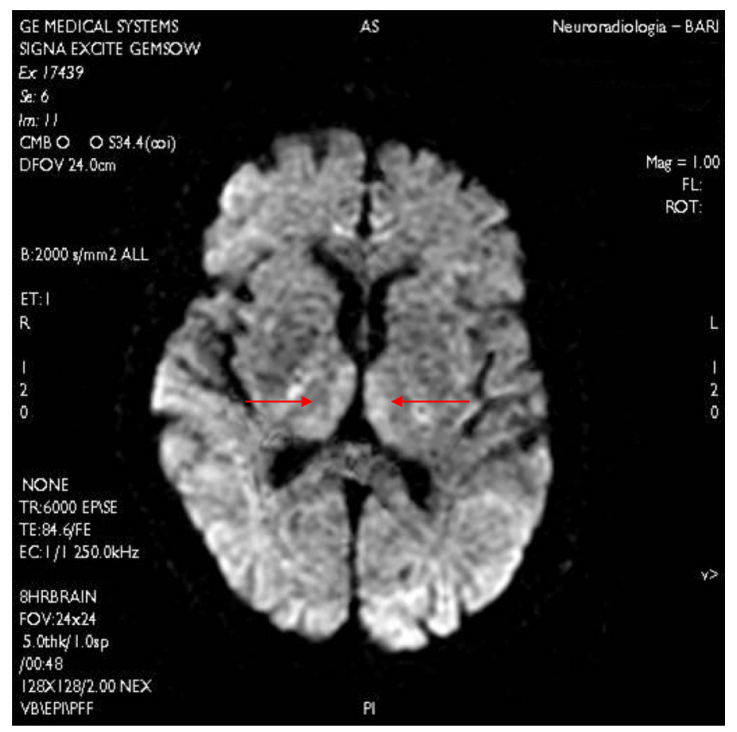

Figure 4 Magnetic resonance imaging: Diffusion-weighted imaging. Symmetrical hyperintense lesions in the medial thalami.

thiamine are only sufficient for up to 18 days. A primary and a secondary deficit of Thiamine are recognized. The former is caused by a bad diet: for example among people who eat only polished rice. ${ }^{6}$ The second is caused by an increased demand, as occurs in cases of hyperthyroidism, during pregnancy, breast-feeding, and fever; or due to defective assimilation in cases of recurrent vomiting or protracted diarrhea; or to altered assimilation as in the case of serious liver dysfunctions. In the case of alcoholism, for example, the condition is due to a combination of reduced thiamine assimilation, altered absorption and usage, and an increased need for this substance.

A thiamine deficiency should be suspected in patients with hyperemesis, ${ }^{7}$ starvation, cancer, acquired immune deficiency syndrome (AIDS), chronic alcoholism and undergoing dialysis, gastroplasty, or gastric bypass surgery. The diagnosis can be made on the basis of vomiting, lateral or medial straight muscle paralysis that causes uni- or bilateral ophthalmoplegia, fever, asthenia, ataxia, precordial pain, anorexia, constipation, irritability, loss of memory, insomnia, and a general degeneration of the mental faculties that usually evolves into a general confusional state, coma and, ultimately death.

Differential diagnosis must include hypophosphatemia, Miller-Fischer's syndrome, and central pontine myelinolysis. Weakness of skeletal or smooth muscle is the most common clinical manifestation of phosphate deficiency. It can involve any muscle group, alone or in combination, ranging from ophthalmoplegia to proximal myopathy to dysphagia or ileus. Mental status abnormalities may occur, with severe hypophosphatemia, ranging from simple irritability or confusion to a severely altered mental status and coma. Hypophosphatemia 
is frequently due to chronic alcoholism, chronic ingestion of phosphate-binding antacids, parenteral nutrition, or refeeding after prolonged starvation. This diagnosis was excluded in the presented case report by the normal plasma phosphate levels: $3.1 \mathrm{mg} / \mathrm{dL}$ (normal range $2.5-4.5 \mathrm{mg} / \mathrm{dL}$ ).

Central pontine myelinolysis is concentrated, frequently symmetric, noninflammatory demyelination within the central basis of the pons. The precise mechanism that strips the myelin sheath is unknown. Central pontine myelinolysis is a disease predominantly observed in alcoholics but also in non-alcoholics with liver diseases, malnutrition, cancer, congestive heart failure, adrenal insufficiency, and renal disease. ${ }^{8}$ The most common cause is a rapid correction of hyponatremia. The symptoms include weakness of the extremities, diplopia, dysarthria, changes in corticospinal reflexes, dysphagia, and confusion. ${ }^{9}$ This diagnosis was excluded by the almost normal plasma sodium concentration (134 mM, normal range: 136-146 mM).

Miller-Fischer's syndrome is a rare variant of GuillainBarré syndrome and manifests as a descending paralysis, proceeding in reverse order as compared to the more common form of Guillain-Barré syndrome. It usually affects the eye muscles first and presents with the triad ophthalmoplegia, ataxia, and areflexia. Anti-GQ1b antibodies are present in $90 \%$ of cases, ${ }^{10}$ but weakness and sensory disturbances of the limbs may also occur. The syndrome is associated with upper respiratory tract infection. All forms of GBS are due to an immune response to foreign antigens (such as infectious agents) that are mistargeted at host nerve tissues instead. The targets of such an immune attack are thought to be the gangliosides, compounds that are naturally present in large quantities in human nerve tissues. Our patient had the classical triad of MFS, but no history of infection. Furthermore, her full recovery after treatment with thiamine ruled out this diagnosis.

Among instrumental exams, magnetic resonance imaging is the gold standard to diagnose Wernicke's encephalopathy. Brain MRI shows symmetrical hyperintense lesions in the medial thalami, in periaqueductal regions of the mid brain and mammillary bodies.
The development of Wernicke's encephalopathy in our patient was due to persistent vomiting and malnutrition secondary to hypoadrenalism, which was caused by the sudden suspension of prolonged steroidal therapy. Complete recovery occurred $24 \mathrm{~h}$ after treatment with adequate doses of thiamine, which confirmed the accuracy of the diagnosis.

In conclusion, we can say that Wernicke's encephalopathy must be viewed as a medical emergency that should be suspected in all those cases showing a sudden deficit of VI cranial nerve function or lack of coordination of the ocular movements associated with ataxia and a general degeneration of mental faculties, that usually evolves into a general confusional state, in patients who have suffered malnutrition or recurrent vomiting for different reasons.

\section{Disclosure}

The authors affirm that they have no financial relationship or conflict of interest with any biotechnology manufacturer that has an interest in the subject matter or materials discussed in the submitted manuscript.

\section{References}

1. Zubaran C, Fernandes JG, Rodnight R. Wernicke-Korsakoff syndrome. Postgrad Med J. 1997;73:27-31.

2. D'Aprile P, Gentile MA, Carella A. Enhanced MR in the acute phase of Wernicke encephalopathy. AJNR Am J Neuroradiol. 1994;15:591-593.

3. Gallucci M, Bozzao A, Splendiani A, et al. Wernicke encephalopathy: MR findings in five patients. AJNR Am J Neuroradiol. 1990;11:887-892.

4. Nolli M, Barbieri A, Pinna C, Pasetto A, Nicosia F. Wernicke's encephalopathy in a malnourished surgical patient: clinical features and magnetic resonance imaging. Acta Anaesthesiol Scand. 2005 Nov;49:1566-1570.

5. Krill JJ. Neuropathology of thiamine deficiency disorders. Metab Brain Dis. 1996;11:9-17.

6. Victor M, Adams R, Collins G. The Wernicke-Korsakoff syndrome and related neurologic disorders due to alcoholism and malnutrition. 2nd ed. 1989. Philadelphia, PA: FA Davis.

7. Fischer J, Müller A, Pohl C, Jens H. Bedrohlicher thiaminmangel bei schwerer hyperemesis gravidarum. Schweiz Med Wochenschr. 1993;123:428-431.

8. Ashrafian H, Davey P. A review of the causes of central pontine myelinosis: yet another apoptotic illness? Eur J Neurol. 2001 Mar; 8(2):103-109.

9. Charness ME. Brain lesions in alcoholics. Alcohol Clin Exp Res. 1993 Feb; 17(1):2-11.

10. Snyder LA, Rismondo V, Miller NR. The Fischer variant of GuillainBarré syndrome (Fischer syndrome). J Neuroophthalmol. 2009 Dec; 29(4):312-324.
Pathology and Laboratory Medicine International

\section{Publish your work in this journal}

Pathology and Laboratory Medicine International is a peer-reviewed, open access journal focusing on innovative basic research and translational research related to pathology or human disease. The journal includes original research, updates, case reports, reviews and commentaries on current controversies. The Academic Sponsor

\section{Dovepress}

of this journal is the Chinese American Pathology Association (CAPA). The manuscript management system is completely online and includes a very quick and fair peer-review system. Visit http://www.dovepress.com/testimonials.php to read real quotes from published authors.

Submit your manuscript here: http://www.dovepress.com/pathology-and-laboratory-medicine-international-journal 